\title{
Análisis del discurso: de la lexicometría a la fraseología. El caso de los sintagmas nominales en el discurso político colombiano
}

\author{
Análise do discurso: da lexicometria à fraseologia. O caso dos sintagmas nominais no \\ discurso político colombiano
}

\author{
Henry Hernandez Bayter ${ }^{1}$ \\ Université de Lille
}

RESUMEN: Nos proponemos aquí estudiar y analizar con la ayuda de un programa lexicométrico, Lexico 5, los dos textos del acuerdo de paz en Colombia. Nuestro objetivo es poner de manifiesto los diferentes sintagmas nominales que se constituyen y fijan en nuestro corpus de estudio y las estrategias discursivas que se construyen alrededor de ellos. El estudio se centra, por consiguiente, en las formas polilexicales nominales utilizadas en cada texto y en los diferentes campos semánticos que se construyen y gravitan en torno a dichas formas. Se utiliza así los diferentes métodos lexicométricos para identificar las formas o nodos monolexicales que se constituyen en temáticas. Más adelante se explotan el análisis distribucional y las concordancias para extraer las unidades polilexicales y las coocurrencias que forman un contexto frástico que sirve a la construcción de una estrategia discursiva en cada texto. La primera fase nos permitió identificar la forma o nodo "organizaciones" y la segunda fase nos permitió estudiar las formas polilexicales "organizaciones criminales", "organizaciones de mujeres", "organizaciones basadas en la fe", entre otras. Comparando los dos textos, pudimos identificar una estrategia de atenuación de la controversia que se creo alrededor del primer texto y de las diferentes designaciones utilizadas para referirse a los participantes del acuerdo.

Palabras claves: Análisis del discurso; Fraseología; Lexicometría; Sintagmas nominales; Formas polilexicas.

RESUMO: Propomos aqui estudar e analisar, com a ajuda de um programa lexicométrico, os dois textos do acordo de paz na Colômbia. Nosso objetivo é destacar os diferentes sintagmas nominais que são constituídos e fixados em nosso corpus de estudo e as estratégias discursivas que são construídas em torno deles. O estudo centra-se, portanto, nas formas poliléxicas nominais utilizadas em cada texto e nos diferentes campos semânticos que são construídos e gravitam em torno dessas formas. Os diferentes métodos lexicométricos são assim utilizados para identificar as formas ou nós monolexicais que se constituem nos temas. Posteriormente, a análise distribucional e as concordâncias são exploradas para extrair as unidades polilexicais e as coocorrências que formam um contexto frástico que serve para a construção de uma estratégia discursiva em cada texto. A primeira fase nos permitiu identificar a forma ou nó "organizações" e a segunda fase nos permitiu estudar as formas polilexicais "organizações criminosas", "organizações de mulheres", "organizações baseadas na fé", entre outras. Ao comparar os dois textos, foi possível identificar uma estratégia para mitigar a

\footnotetext{
${ }^{1}$ Doctor en lingüística del español. Facultad de Lenguas Aplicadas de la Universidad de Lille. Laboratorio de investigación STL.
} 
controvérsia que foi criada em torno do primeiro texto e as diferentes designações utilizadas para se referir aos participantes no acordo.

Palavras-chave: Análise do discurso; Fraseologia; Lexicometria; Sintagmas nominais; Formas poliléxicas. 
Henry Hernandez Bayter. Análisis del discurso: de la lexicometría a la faseología. El caso de los sintagmas nominales en el discurso político colombiano.

El presente artículo tiene como objetivo la exploración y ejemplificación de una metodología cuantitativa aplicada a la teoría de la fraseología y a la del análisis del discurso. Tomaremos como punto de partida algunas unidades polilexicales extraídas de un corpus de estudio compuesto por los dos textos del acuerdo de paz en Colombia. Teniendo en cuenta las teorías del análisis del discurso y de la fraseología, con la ayuda de una metodología de exploración y estadística textual, tratamos poner en evidencia la presencia de ciertas unidades polilexicales que relevan de los sintagmas nominales o sustantivos compuestos que se forman al interior de dicho corpus. Tratamos entender como estas unidades multipalabra son utilizadas al interior de los dos textos con el fin de servir de base a diferentes estrategias discursivas. De esta manera, analizamos unidades que constituyen secuencias que se fijan y hacen referencia a ciertos aspectos discursivos particulares y relevantes del corpus de estudio.

\section{Contextualización y elección del corpus}

Para nuestro análisis, hemos decidido utilizar los dos textos del acuerdo de paz, lo cual no permite respetar las condiciones de todo estudio lexicométrico: la comparabilidad y la coherencia de los textos que componen el corpus.

Para entender mejor la elección del corpus, proponemos aquí una breve contextualización de los dos textos. Dicha contextualización no busca, en ningún caso, ser exhaustiva, sino, por el contrario, representativa de manera breve de la situación de producción de dichos textos.

De manera cronológica, la Corte Constitucional de Colombia aprueba el plebiscito el 18 de julio de 2016 que seria una consulta hecha al conjunto de la población colombiana tras el fin de los diálogos entre el gobierno de Juan Manuel Santos y las Fuerzas Armadas Revolucionarias de Colombia (FARC). El 24 de agosto de 2016, se anuncia el fin de las conversaciones en la Habana y se prepara el primer texto que será sometido a la opinión pública el 2 de octubre del mismo año. Para la aprobación o el rechazo de dicho texto, la población tendría que expresar su acuerdo o su desacuerdo con el mismo, respondiendo si o no a la siguiente pregunta: «iApoya el acuerdo final para la terminación del conflicto y construcción de una paz estable y duradera?».

La consulta arroja como resultado la victoria del "No" con un total de 50,21\% contra $49,78 \%$ del "Si". La votación se ve enmarcada por una tasa de abstención bastante alta: $62,59 \%$. Un segundo texto es ratificado y aprobado el 24 de noviembre de 2016, poniendo así 
Henry Hernandez Bayter. Análisis del discurso: de la lexicometría a la faseología. El caso de los sintagmas nominales en el discurso político colombiano.

fin a la existencia de las FARC como grupo guerrillero y al nacimiento de las FARC como partido político, pero también acabando con uno de los grupos guerrilleros más antiguos de la historia colombiana.

Los detractores del texto se aferraron a las 111 coocurrencias de la palabra "género" y rápidamente asociaron dicha palabra al sintagma nominal "ideología de género". Mientras que los partidarios del texto asociaban esta palabra a otro sintagma nominal "equidad de género" y explicaban que el énfasis se hacia en el trato igualitario entre hombres y mujeres dentro del conflicto armado.

Esta guerra de las palabras y de los singtamas nominales motivó nuestro estudio. Decidimos explorar de dicha forma los dos textos para tratar de entender y explorar los diferentes sintagmas nominales que se fijan al interior de los dos textos y que relevan de una cierta estrategia fraseológica por medio del discurso y como apoyo de unas estrategias discursivas dadas.

\title{
Marco teórico
}

\begin{abstract}
Pour analyser un phénomène social, il convient de dire de quel point de vue on le fait, c'est-à-dire dans quelle discipline des sciences humaines et sociales on se situe, et, à l'intérieur de celle-ci, quelle orientation l'on suit (CHARAUDEAU, 2011, p. 105).
\end{abstract}

Como precisa Charaudau, para poder pasar al análisis en concreto de los dos textos como fenómeno social colombiano, tendremos que establecer un marco teórico en el cual nos basaremos y que nos permitirá guiar nuestra reflexión. Dicho marco teórico nos llevará de manera concatenada a la metodología utilizada y al interés de los métodos utilizados en el programa computacional utilizado de lexicometría, Lexico 5, que presentaremos detalladamente más adelante.

Nos posicionaremos de esta manera en el marco de los estudios del análisis del discurso propuestos por Charaudeau, Fernández Lagunilla y Maingueneau, entre otros. Así, para el análisis del discurso político nos restringiremos al análisis de la estructura de este, como precisa Fernández Lagunilla (1999): quién habla, a quién se habla y sobre qué se habla. Se tiene en cuenta tanto el locutor, como los interlocutores y el mensaje que se transmite por medio del discurso, en una situación particular. En nuestro caso, esta situación corresponde a un dispositivo textual, los dos textos del acuerdo de paz, en el cual intervienen los actores de 
Henry Hernandez Bayter. Análisis del discurso: de la lexicometría a la faseología. El caso de los sintagmas nominales en el discurso político colombiano.

la vida política colombiana: el presidente Santos y su gobierno, las FARC, las víctimas y los colombianos, en general.

Se sabe igualmente que el discurso político representa un terreno propicio para la implementación de diferentes estrategias discursivas. Para nuestro estudio, nos focalizaremos en el uso de ciertas unidades polilexicales del ámbito de la fraseología en dichos textos como punto de partida para la construcción de estrategias discursivas en ambos textos.

Para nuestra investigación, la fraseología se define como un conjunto de expresiones que contienen ciertas características particulares al nivel de la forma (polilexicalidad, opacidad semántica, entre otras) y propias a un contexto de utilización, también particular. Cabe aclarar que para nuestro estudio hemos utilizado la definición más amplia de los estudios fraseológicos y desde un punto de vista discursivo y no estructural. Por consiguiente, diferimos de los estudios fraseológicos que se encargan de la descripción de la estructura y desde un punto de vista de la lengua de las unidades de este campo. Nuestra definición remite a la existencia de un perfil fraseológico, correspondiente a los contextos y ámbitos de utilización de dichas unidades polilexicales. El perfil fraseológico corresponde a la descripción y al análisis de las diferentes estrategias empleadas en una tipología textual por parte del o de los locutores y que contienen dichas unidades del campo de la fraseología. La identificación de un perfil fraseológico permite poner en evidencia la tipología a la cual corresponde el texto, pero igualmente remite al estilo del locutor y el rol que dicho locutor otorga dichas unidades en el discurso.

\section{Aproximación metodológica adoptada}

Hemos recurrido a la utilización de una metodología cuantitativa que nos permita tener una base consecutivamente objetiva, a partir del recuento estadístico del léxico de los textos de nuestro corpus. Esta primera fase nos conllevará a una segunda fase más cualitativa del análisis de dicho léxico y de su disposición en el contexto textual. El uso del paquete informático de estadística textual, Lexico 5 - programa de estadística textual concebido y desarrollado por André Salem en el laboratorio SYLED CLA2T (Système Linguistique Énonciation Discursivité - Centre d'Analyse Automatique des Textes) de la Universidad Paris 3 , permite poder relacionar los dos textos del tratado de paz y hacer un inventario de las formas más utilizadas, las formas que aparecen con frecuencia en colocación formando unidades polilexicales, de tipo substantívale en nuestro caso, que se fijan al interior del 
corpus. Del mismo modo, el programa permite identificar y caracterizar cada versión del texto.

Varios métodos lexicométricos (SALEM, 1998, p. 6) dan la posibilidad al investigador de referenciar las unidades simples y polilexicales, dejando de lado toda subjetividad. Se parte del recuento de elementos lingüísticos, para luego, en segundo lugar, proceder a un análisis cualitativo de los mismos, de su uso y de su contexto textual y sociopolítico. Según indican Bonafous y Tournier :

Lexicométrie [...] elle est chargée d'examiner, à partir d'un corpus de textes soumis à comparaison, comment les termes échangés dans l'espace public autour des enjeux de pouvoir rendent compte des luttes d'appropriation ou de dépossession symboliques qui se jouent dans le lieu même de l'échange (BONAFOUS; TOURNIER, 1995, p. 69).

De esta forma, el recurrir a la lexicometría permite explorar los desafíos sociales a partir del léxico mismo y de las diferentes unidades tanto simples como polilexicas que aparecen y que son utilizadas de manera estratégica en un texto.

\section{Aproximación lexicométrica del corpus}

Une partition d'un corpus de textes est une division d'un corpus en parties constituées par des fragments de texte consécutifs, n'ayant pas d'intersection commune et dont la réunion est égale au corpus (SALEM et al., 2009, p. 6).

Una primera aproximación lexicométrica del corpus se puede hacer a través las particiones que lo componen. Como podemos ver en el Cuadro 1, aquí abajo, el corpus está compuesto de dos textos y, por lo ende, dos particiones. Una partición corresponde, como indica Salem et al., a cada una de las partes que forman el conjunto del corpus. En el caso del corpus para este estudio, las particiones corresponden a los dos documentos del acuerdo de paz. Esta única partición nos permitirá contrastar los varios apuestos lexicológicos y estructurales de las unidades polilexicales de cada texto. Si analizamos el cuadro, las dos particiones contienen un numero casi idéntico de formas, lo que garantiza la comparación con Lexico 5 se aplica de manera estricta en los estudios lexicométricos. Por el contrario, si miramos el número de ocurrencias, a saber, el número de veces que una forma aparece en un texto, por consiguiente, su frecuencia de utilización, el número de ocurrencias nos permite 
comprender que la segunda versión del acuerdo de paz, retoma más fácilmente el vocabulario empleado, dando la impresión que se presta más a la construcción de sintagmas o unidades polilexicales que se van a fijar en el corpus, gracias a la repetición de las mismas.

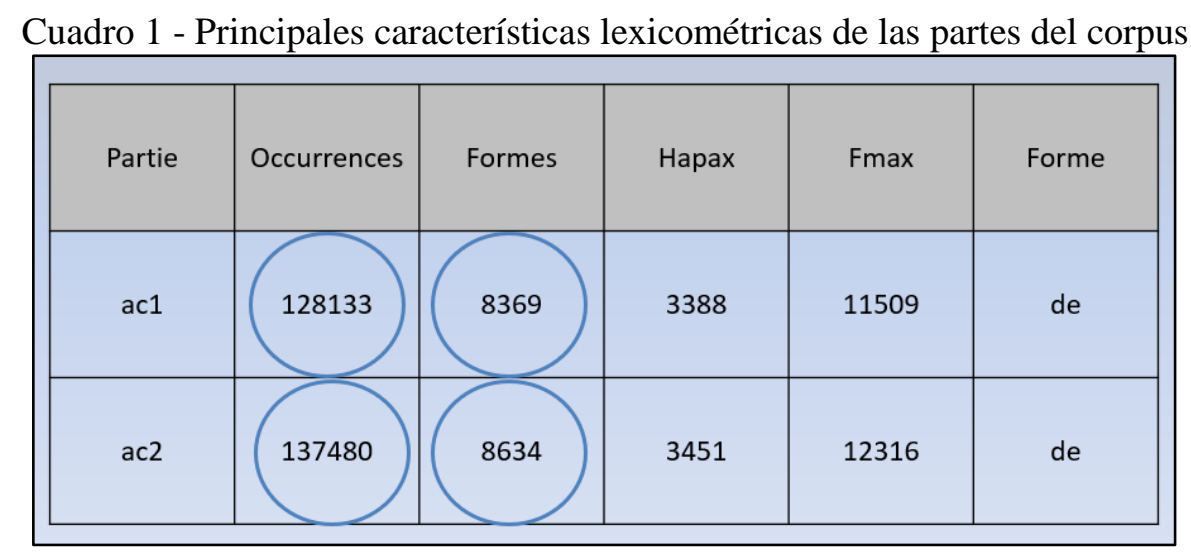

\section{Localización y análisis de las unidades polilexicales}

Para la localización de las unidades polilexicales, hemos decidido explorar el corpus a partir de las unidades simples más frecuentes. Decidimos dejar de lado todas las formas léxicas que hacen referencia a los participantes del acuerdo de paz, a aquellas que se refieren directamente al acuerdo en sí. Nuestra atención se focalizó sobre una forma léxica que en el índice de formas o diccionario posee una frecuencia bastante elevada: "organizaciones", como se puede apreciar en el Cuadro 2 aquí abajo.

Cuadro 2 - Extracto de las condordancias: corpus completo

\begin{tabular}{|c|c|c|c|}
\hline Formes (ordre lexicographique) & Fréquence & Formes (ordre lexicographique) & Fréquence \\
\hline de & 23825 & 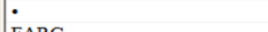 & 1483 \\
\hline y & 12897 & FARC & 1210 \\
\hline la & 12497 & EP & 1208 \\
\hline las & 7310 & un & 1165 \\
\hline el & 7207 & al & 1121 \\
\hline en & 6464 & como & 1062 \\
\hline los & 5829 & El & 1032 \\
\hline a & 4858 & su & 1028 \\
\hline que & 4575 & una & 1011 \\
\hline del & 4508 & Gobierno & 1009 \\
\hline para & 3558 & Nacional & 1002 \\
\hline con & 2525 & Acuerdo & 921 \\
\hline se & 2420 & no & 866 \\
\hline por & 1973 & lo & 851 \\
\hline$\circ$ & 1928 & La & 837 \\
\hline - & 1483 & 1 & 742 \\
\hline FARC & 1210 & sus & 741 \\
\hline EP & 1208 & $\mathrm{v}$ & 703 \\
\hline un & 1165 & MM & 701 \\
\hline al & 1121 & derechos & 647 \\
\hline como & 1062 & $\mathrm{Paz}$ & 631 \\
\hline El & 1032 & organizaciones & 625 \\
\hline su & 1028 & confificto & 608 \\
\hline una & 1011 & 3 & 590 \\
\hline Gobierno & 1009 & En & 588 \\
\hline
\end{tabular}


Henry Hernandez Bayter. Análisis del discurso: de la lexicometría a la faseología. El caso de los sintagmas nominales en el discurso político colombiano.

Como su nombre lo indica, el índice de formas o el diccionario del corpus representa una lista decreciente de la frecuencia de las formas más utilizadas y más representativas del corpus. Dichas formas pueden, en cierta medida, corresponder a las temáticas principales del discurso de cada locutor, en nuestro caso a las temáticas o estrategias discursivas de los dos textos que comparamos. Estas formas-temáticas representan un primer paso para todo análisis discursivo. Ciertas formas corresponden a indicadores del discurso de un locutor, de sus ideas, su ideología y sobre todo de la sociedad en la cual todos estos aspectos aparecen o la tipología textual y a sus características y las estrategias discursivas implementadas en su interior.

Ahora bien, decidimos explorar los sintagmas que se forman y se fijan en el corpus alrededor del nodo o forma base "organizaciones". La elección no es banal. El discurso político colombiano se encuentra poblado de este tipo de formas de designación que se quieren "no evaluativas" como las llama Y. Serrano: "Désignations «non-évaluatives » n'importe quel référent armé légal ou non" (SERRANO, 2012, p. 92). Estas designaciones hacen referencia a los diferentes actores armados del conflicto colombiano, que se trate de grupos legales o ilegales.

Para poder localizar y explorar las secuencias recurrentes y polilexicales que se forman en torno al nodo o forma base "organizaciones", hemos recurrido al método lexicométrico del Inventario de Segmentos Repetidos (SR), propuesto por Lexico 5. Dicho método permite, como afirman Lebart y Salem, poner de manifiesto ciertos grupos de formas que aparecen frecuentemente juntas en el corpus. El empleo de este método nos permite, de forma objetiva y, basándonos en el cálculo de las informaciones textuales, saber que hay ciertos grupos que se fijan a lo largo del corpus debido a su frecuencia de empleo. $\mathrm{Si}$ analizamos el Inventario de Segmentos Repetidos obtenido a partir de Lexico 5, podemos evidenciar una frecuencia de utilización importante y, sobre todo, la característica polilexical de la estructura. Nos ocuparemos aquí de los sintagmas nominales recurrentes en torno entonces al nodo "organizaciones".

Para nuestro estudio presentaremos aquí también un extracto del análisis distribucional del nodo "organizaciones" que también pone de relieve los diferentes sintagmas nominales que se construyen en el corpus y que se repiten frecuentemente y se fijan. 
Henry Hernandez Bayter. Análisis del discurso: de la lexicometría a la faseología. El caso de los sintagmas nominales en el discurso político colombiano.

Cuadro 3 - Extracto del análisis distribucional del nodo "organizaciones"

\begin{tabular}{|c|c|c|}
\hline $3----$ & organizaciones & $\begin{array}{l}\text { basadas en la fe y organizaciones del sector } \\
\text { religioso }\end{array}$ \\
\hline 4-ーーーーーーーーーーーーーーーーーーーー & organizaciones & campesinas \\
\hline 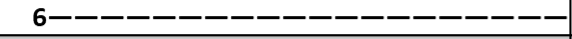 & organizaciones & comunitarias \\
\hline 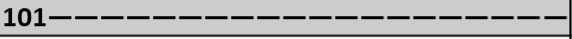 & organizaciones & criminales \\
\hline $8--------$ & organizaciones & criminales dedicadas al narcotráfico \\
\hline $14----$ & organizaciones & criminales objeto de este acuerdo \\
\hline 21-ーーーーーーーーー-ー & organizaciones & de derechos humanos \\
\hline 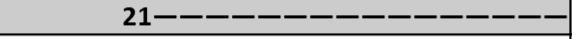 & organizaciones & de mujeres \\
\hline 44-ーーーーーーーーーーーー-ー- & organizaciones & de víctimas \\
\hline $6----------\cdots$ & organizaciones & del sector religioso \\
\hline 7ーーーーーーーーーーーーーーーーーーー- & organizaciones & especializadas \\
\hline 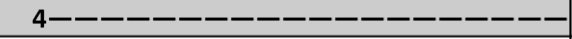 & organizaciones & internacionales \\
\hline $4--------$ & organizaciones & nacionales e internacionales especializadas \\
\hline $6----------------$ & organizaciones & no gubernamentales \\
\hline 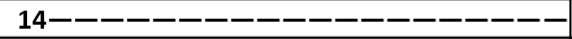 & organizaciones & representativas \\
\hline $6----$ & organizaciones & representativas de los pueblos étnicos \\
\hline 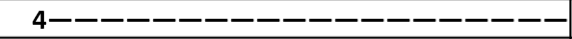 & organizaciones & rurales \\
\hline 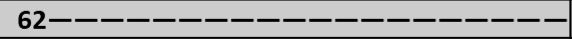 & organizaciones & sociales \\
\hline 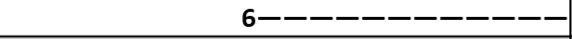 & organizaciones & sociales y comunitarias \\
\hline 84-ーー-ー-ー-ー-ー- & organizaciones & y movimientos sociales \\
\hline $10----$ & organizaciones & y movimientos sociales más representativos \\
\hline
\end{tabular}

El análisis distribucional, método lexicométrico, permite visualizar en un cuadro:

- La frecuencia de aparición del sintagma nominal en el corpus.

- El nodo o forma base.

- Las formas que vienen a aparecer colocadas a la forma nodo.

Encontramos así ciertas colocaciones de tipo Sustantivo + Sustantivo que se encuentra en coocurrencia y en forma binaria. Estas constituyen un bloque completo que contiene un cierto significado, menos opaco que el de las expresiones idiomáticas y que los proverbios. Dichos bloques obtienen un significado particular dentro del corpus en el que se encuentren.

Dentro de las designaciones no-evaluativas y que constituyen un sintagma nominal que se fija en el corpus podemos encontrar: "organizaciones criminales". Dicha designación no-evaluativa sustituyó en el discurso político colombiano otras designaciones también polilexicales que estaban completamente cargadas de connotación negativa. "Organizaciones criminales" busca matizar en el acuerdo de paz un discurso bélico que se había ido construyendo durante mucho tiempo en la sociedad colombiana. Así, este sintagma nominal remplazó dentro del acuerdo a: "grupos guerrilleros", "bandas criminales", "bacrim", "paramilitares", entre otras. Además, el sintagma nominal se ve acompañado de "objeto de este acuerdo" lo cual refleja una estrategia de atenuación por medio de una designación noevaluativa para evitar que dentro del acuerdo de paz se encuentre un léxico y sintagmas que hacen referencia al discurso político del expresidente Álvaro Uribe Vélez. Sin embargo, encontramos un contexto frástico que diferencia las organizaciones criminales que son el 
Henry Hernandez Bayter. Análisis del discurso: de la lexicometría a la faseología. El caso de los sintagmas nominales en el discurso político colombiano.

objeto del acuerdo y que, por consiguiente, se someten a dicho texto y aquellas organizaciones criminales que no entran en el acuerdo y que se dedican al trafico de drogas. ¿Se trata en ese caso de hacer referencia a los grupos disidentes de las FARC que no deseaban someterse al acuerdo? En cualquier caso, podemos ver que la estrategia discursiva es de atenuar el discurso por medio de designaciones no-evaluativas y además, y mucho mas importante, diferenciar las organizaciones que hacen parte y las que no del acuerdo.

Si exploramos las coocurrencias del mismo nodo, encontramos bajo forma de grafico el mismo contenido y relación lexical que se encuentra en el análisis distribucional. Así podemos ver el campo léxico que se va tejiendo a partir del nodo "organizaciones". Como explica Maingueneau :

L'étude des co-occurrences d'un pôle permet de comparer l'entourage lexical d'une forme ... dans le corpus fonctionnant à l'intérieur du même corpus (on débouche ainsi sur une approche intéressante de la notion de "synonymie") (MAINGUENEAU, 1991, p. 61).

La figura que se obtiene es la siguiente:

Figura 1 - Gráfico de las coocurrencias de la forma "organizaciones"

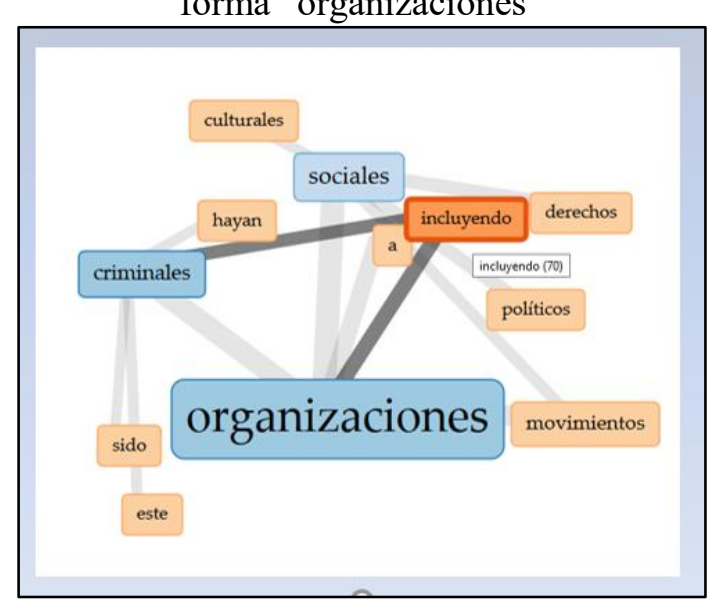

Esta forma-base corresponde al centro de un campo semántico específico alrededor del cual gravitan otras formas que se integran y que buscan reforzar la temática. Las coocurrencias nos permiten poner de realce que éstas aparecen en un contexto preciso y con una utilización precisa en el discurso. En la figura podemos percibir: organizaciones criminales, organizaciones sociales, organizaciones incluyendo, entre otras combinaciones posibles de sintagmas. 
Henry Hernandez Bayter. Análisis del discurso: de la lexicometría a la faseología. El caso de los sintagmas nominales en el discurso político colombiano.

Nos concentraremos en lo que sigue en un sintagma nominal que, a primera vista, pasa desapercibido. Se trata del sintagma nominal "organizaciones de mujeres" que tiene una frecuencia de 21 ocurrencias y que si alargamos el contexto descubrimos que dicho sintagma va acompañado de otras formas léxicas del corpus "LGBTI" y de sintagmas nominales "diversidad sexual". De esta forma, los dos sintagmas nominales en el primer texto del acuerdo se encuentran en el siguiente contexto frástico "organizaciones de mujeres y LGBTI" y "organizaciones de mujeres y diversidad sexual". Ambas hacen referencia a la polémica que fue desatada por los opositores del primer texto del acuerdo que afirmaban que el acuerdo era una apología a la "ideología de género" confundiendo así el objetivo principal del acuerdo que era crear una equidad de género, tanto dentro de las victimas como dentro de los guerrilleros que se acogían al acuerdo. Esta controversia fue la hoja de ruta de la oposición para las modificaciones que habría que efectuar al texto tras el plebiscito.

Mientras que, en el segundo texto, el mismo sintagma no tiene ninguna frecuencia de aparición, incluso no aparece como hápax (única ocurrencia de una forma en el corpus). En lexicometría, una forma o un conjunto de formas que se sueldan y forman sintagmas nominales fijos en el corpus no desaparecen, sino que son remplazadas por otras formas o sintagmas que dan un viraje a la estrategia discursiva que se desea construir. En su lugar, encontramos "organizaciones basadas en la fe" y "organizaciones el sector religioso". Para evitar la controversia, el texto es modificado y toda alusión a las organizaciones compuestas por mujeres o que hagan referencia a la equidad de sexos es evitada. Se busca así atenuar el texto del acuerdo de paz por medio de una estrategia discursiva. Se remplaza un sintagma nominal que se había fijado en el primer texto por un nuevo sintagma nominal que se fija en el segundo texto. Dicho sintagma hace referencia a la religión y a las creencias comunes de los colombianos. Así se vuelve a un léxico conservador que hace eco en la memoria colectiva de los colombianos y sus creencias religiosas. Dicha estrategia de utilización de unidades léxicas que hacen referencia a la vida religiosa, ya había sido estudiada en los discursos del expresidente Álvaro Uribe Vélez. Se trata así de establecer una conexión, un puente de relación estrecha entre las creencias de los colombianos y lo estipulado en el segundo texto del acuerdo, lo que busca dar una efectividad y rapidez a la transmisión del mensaje contenido en este nuevo acuerdo y evitar, a todo precio, una nueva reacción negativa del texto.

Para concluir nuestro estudio podemos afirmar que el estudio y análisis de los dos textos del acuerdo de paz en Colombia, presentado aquí, desde el punto de vista del análisis del discurso estudiado en Francia y de la fraseología y por medio de una herramienta informática de tipo cuantitativo, permite la exploración de manera objetiva de los diferentes 
Henry Hernandez Bayter. Análisis del discurso: de la lexicometría a la faseología. El caso de los sintagmas nominales en el discurso político colombiano.

elementos lingüístico-discursivos utilizados en ambos textos con el fin de construir estrategias discursivas.

La aproximación lexicométrica permite así partir del léxico en si mismo. Para el estudio de las unidades fraseologías o de tipo polilexical, una primera aproximación de estadística textual permite despejar ciertas formas y nodos monolexicales que representan de cierta forma temáticas o formas indispensables para la construcción de estrategias discursivas en el interior de cada texto. Por otro lado, el uso de dichas formas simples y de dichos nodos sirven de punto de base para la construcción argumentativa de cada texto.

Por otro lado, la utilización del inventario distribucional, de los segmentos repetidos y de las coocurrencias y sus gráficos, permite poner en evidencia la constitución de sintagmas nominales que se fijan y adquieren cierto significado dentro de cada texto. Además de los sintagmas, dichos métodos lexicométricos permiten la exploración del contexto frástico y de los campos semánticos que se construyen alrededor de ellos.

Así, hemos podido detectar la utilización de la forma simple "organizaciones" con una alta frecuencia en el corpus y explorar los sintagmas nominales que se construyen alrededor de esta forma-nodo. Dicha exploración nos llevo a estudiar los sintagmas "organizaciones criminales" pero del mismo modo nos llevo a estudiar otros campos mas amplios de coocurrencias con "organizaciones de mujeres y LGBTI", entre otras, en el primer texto, y "organizaciones basadas en la fe" en el segundo texto. Así, pudimos estudiar la tan sonada controversia de la "teoría de género" y poner en evidencia la utilización de los aspectos religiosos en la sociedad colombiana como estrategia de captación y de eficacidad del texto.

\section{Referencias}

BONNAFOUS S.; TOURNIER M. Analyse du discours, lexicométrie, communication et politique. Les analyses du discours en France, Langages, 29e année, n. 117, p. 67-81, 1995. Disponible en: <https://www.persee.fr/doc/lgge_0458-726x_1995_num_29_117_1706>.DOI: https://doi.org/10.3406/lgge.1995.1706

CHARAUDEAU, P. Réflexions pour l'analyse du discours populiste, Mots, n. 97, Les collectivités territoriales en quête d'identité. Lyon, ENS Éditions, p. 101-116, 2011. Disponible en: $\quad<$ https://journals.openedition.org/mots/20534>. DOI: https://doi.org/10.4000/mots.20534

CHARAUDEAU, P. La conquête du pourvoir. Opinion, persuasion, valeur. Les discours d'une nouvelle donne politique. Collection Langue\& Parole. Paris: L'Harmattan, 2013. 
CHARAUDEAU, P. A quoi sert d'analyse le discours politique ?, Análisi del discurspolític, IULA-UPF, Barcelone, 2002. Disponible en: <http://www.patrick-charaudeau.com/A-quoisert-d-analyse-le-discours.html>. Consultado el: 26 noviembre 2014.

CHARAUDEAU, P.; MAINGUENEAU, D. Dictionnaire d'analyse du discours. Paris: Éditions du Seuil, 2002.

FERNÁNDEZ LAGUNILLA, M. La lengua en la comunicación política. Tomo I: El discurso del poder. Madrid: Arco Libros, 1999.

FERNÁNDEZ LAGUNILLA, M. La lengua en la comunicación política. Tomo II: La palabra del poder. Madrid: Arco Libros, 1999.

LEBART, L.; SALEM A. Statistique textuelle [En ligne]. Paris: Édition DUNOD, 1994. Disponible en: <http://lexicometrica.univ-paris3.fr/livre/st94/st94-tdm.html>.

MAINGUENEAU, D. Nouvelles tendances en analyse du discours. Paris: Hachette, 1987.

MAINGUENEAU, D. L'analyse du discours. Introduction aux lectures de l'archive. Paris: Hachette, 1991.

SALEM, A. Segments répétés et analyse statistique des données textuelles, Histoire \& Mesure. v. I-II, p. 5-28, 1986. Disponible en: <https://www.persee.fr/doc/hism_09821783_1986_num_1_2_1518>. DOI: https://doi.org/10.3406/hism.1986.1518

SALEM, A. Approches du temps lexical, statistique textuelle et séries chronologiques, Mots, v. 17, p. 105-143, 1998.10 Disponible <https://www.researchgate.net/publication/250245868_Approches_du_temps_lexical_Statisti que_textuelle_et_series_chronologiques >. DOI: https://doi.org/10.3406/mots.1988.1401

SALEM, A. Lexico 3. Explorations textométriques. v. 2: séries textuelles chronologiques. Paris: SYLED-CLA2T, Université de la Sorbonne Nouvelle - Paris 3, 2009.

SALEM, A. et al. Lexico 3. Outils de statistique textuelle. Manuel d'utilisation. Paris: SYLED-CLA2T, Université de la Sorbonne Nouvelle - Paris 3, 2003.

SERRANO, Y. Nommer le conflit armé et ses acteurs en Colombie. Communication ou information médiatique ?. Paris: L'Harmattan, 2012.

Recebido em: 8 de abril de 2019

Aceito em: 14 de junho de 2019 